\title{
Andreev Reflection in Transport Through a Quantum Dot Interacting With a Polaron
}

\author{
K. BOCIAN*, W. RudzińsKI \\ Faculty of Physics, Adam Mickiewicz University, ul. Umultowska 85, 61-614 Poznań, Poland
}

\begin{abstract}
Spin-dependent tunneling through a quantum dot coupled to one ferromagnetic and one superconducting electrodes is studied theoretically in the Andreev reflection (AR) regime. Spectral functions for the system are calculated in terms of the nonequilibrium Green function technique. Effects due to interplay between the Coulomb correlations on the dot and the local phonon mode in the context of the AR transmission are analyzed.
\end{abstract}

DOI: 10.12693/APhysPolA.126.374

PACS: 72.25.Mk; 73.23.Hk; 73.63.Kv; 74.25.F-

\section{Introduction}

Effects due to the Andreev reflection (AR) phenomenon in a hybrid system consisting of ferromagnet $(\mathrm{F})$, quantum dot $(\mathrm{QD})$ and superconductor (S) have been studied recently in a number of papers (see e.g. $[1-5])$. In particular, some attention has been devoted to AR in the presence of electron-phonon interactions [4]. It has been predicted that electron-phonon interactions may suppress the AR current flowing through the junction F-QD-S, and that spin-flip processes on the dot may give rise to splits of the both main as well as the phonon conductance resonances. Up to now, the polaronic transport has been studied for QDs with vanishing charging energy. In this paper we discuss the properties of AR spectral functions for the F-QD-S system with vibrational degrees of freedom, assuming arbitrary Coulomb correlations on the dot.

\section{Model and method}

Consider a single-level QD coupled via tunnel barriers to one ferromagnetic and one superconducting lead. The whole system can be described by Hamiltonian of the general form $H=H_{f}+H_{s}+H_{p h}+H_{d}+H_{t}$. The terms $H_{f}$ and $H_{s}$ describe the ferromagnetic electrode in the non-interacting quasi-particle approximation and the BCS Hamiltonian of the S lead, respectively (for details see e.g. [5]). The third term is the phonon Hamiltonian, $H_{p h}=\omega_{0} b^{+} b$, where $\omega_{0}$ is the vibrational frequency of the phonon mode and $b^{+}(b)$ is the phonon creation (annihilation) operator. The quantum dot is described by $H_{d}=\sum_{\sigma}\left[\varepsilon_{d}+\lambda\left(b+b^{+}\right)\right] d_{\sigma}^{+} d_{\sigma}+U n_{\uparrow} n_{\downarrow}$, where $\varepsilon_{d}$ denotes the energy of the discrete level, $U$ is the electron correlation parameter, the parameter $\lambda$ describes strength of the electron-phonon coupling, while $d_{\sigma}^{+}$and $d_{\sigma}$ are the relevant creation and annihilation operators for spin $\sigma$

\footnotetext{
*corresponding author; e-mail: bocian@amu.edu.pl
}

$(\sigma=\uparrow, \downarrow)$. Finally, the tunnelling term $H_{t}$ describes processes due to coupling of QD to the external leads [5]. Using the Lang-Firsov-type unitary transformation [6], one may eliminate the linear coupling terms in the QD Hamiltonian, leading to renormalization of the dot energy level $\varepsilon_{d}^{\prime}=\varepsilon_{d}-\lambda^{2} / \omega_{0}$, as well as to renormalization of the charging energy $U^{\prime}=U-2 \lambda^{2} / \omega_{0}$. The Lang-Firsov transformation gives rise also to exponential suppression of the tunneling amplitudes in the tunneling term $H_{t}[6]$, which in turn leads to the charge conserving $\lambda$-dependent Franck-Condon blockade of tunneling processes between the dot and an external electrode.

By means of the nonequilibrium Green-function technique in the $4 \times 4$ Nambu representation [2], one may next calculate the spectral functions for Andreev reflection from the formula $A=-(1 / \pi) \operatorname{Im}\left(G_{11}^{R}+G_{33}^{R}\right)$, where $G_{i i}^{R}$ is the retarded Green function of the QD. The coupling strength between the QD and the electrodes is described by hybridization couplings up on which these Green functions depend. The relevant for our further discussion are the quantities $\Gamma_{f \uparrow(\downarrow)}=\Gamma_{f 0}(1 \pm P)$, where $P$ is the corresponding spin polarization factor and $\Gamma_{f 0}$ is a coupling constant for the F-QD junction. The coupling strength between QD and the S electrode is governed by the quantity $\Gamma_{s 0}$, which is responsible for the particle-hole splitting in AR and gives rise to appearance of the Andreev bound states.

\section{Numerical results}

In the following we shall discuss features of the AR spectral function or the density of states (DOS) at zero temperature, assuming $\Gamma_{f 0}<\Gamma_{s 0}$. Energy, temperature as well as the strength of the electron-phonon interaction $\lambda$ is measured relative to the energy gap of the superconducting lead.

The spectral function in Fig. 1 shows that if the Andreev reflection occurs in the presence of the vibrational mode localized on the dot, then besides the main resonances (see inset in Fig. 1a) also phonon sidebands emerge. In case of $\varepsilon_{d}^{\prime}=U^{\prime}=0$ (Fig. 1a) the emission phonon satellites are separated from each other by 


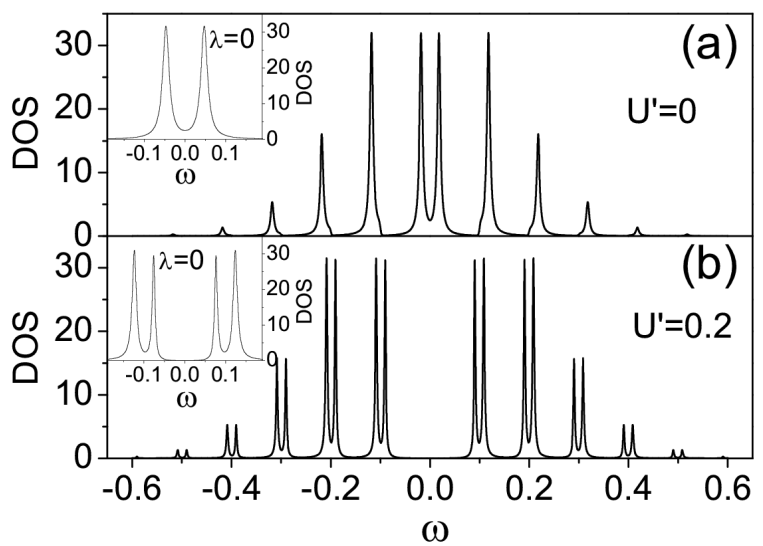

Fig. 1. Density of states calculated for $\varepsilon_{d}^{\prime}=0$ with $U^{\prime}=0(\mathrm{a})$ and $\varepsilon_{d}^{\prime}=-0.1$ with $U^{\prime}=0.2(\mathrm{~b})$. The other parameters are: $\lambda=0.1, \omega_{0}=0.1, T=0, P=$ $0.5, \Gamma_{f 0}=0.02$ and $\Gamma_{s 0}=0.1$. The insets show the corresponding density of states obtained for vanishing electron-phonon interactions, $\lambda=0$.

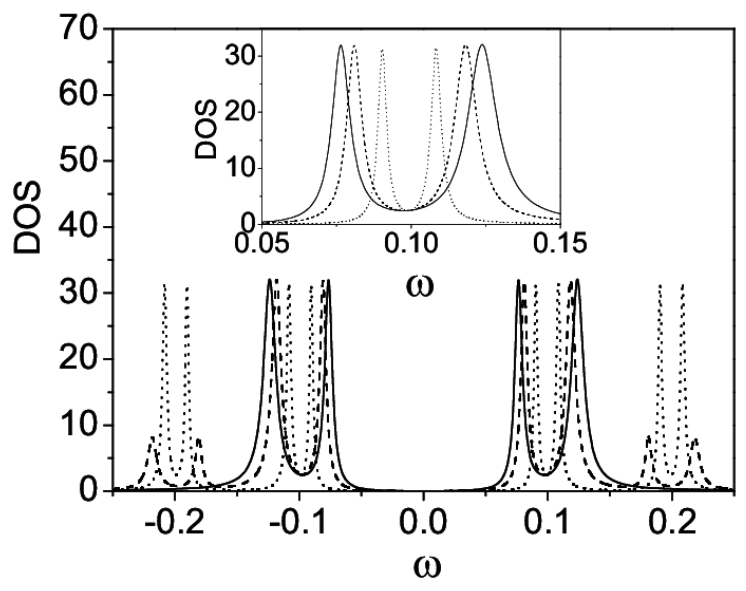

Fig. 2. Density of states calculated for $\varepsilon_{d}^{\prime}=-0.1$ with $U^{\prime}=0.2$ and $\lambda=0$ (solid line), $\lambda=0.05$ (dashed line) and $\lambda=0.1$ (dotted line). The other parameters are as in Fig. 1. The inset shows in more detail the $\lambda$ dependent renormalization of the Andreev bound sates formed around the energy $\varepsilon_{d}^{\prime}+U^{\prime}$.

the phonon energy and appear on one side of the corresponding Andreev bound state. The DOS intensity for carriers transmitted through the in-gap QD states in the presence of the local phonon mode is suppressed with increasing $\omega$. If $U^{\prime}>0$ and $\lambda=0$, then for the electronhole symmetry, $\varepsilon_{d}^{\prime}=-U^{\prime} / 2$, the spectral function exhibits two resonances at $\pm U^{\prime} / 2$. Each of these resonance peaks is additionally split due to transmission through the Andreev bound states (see inset in Fig. 1b). One pair of DOS maxima appears for the single occupied dot, while the other pair appears for the dot occupied by two carriers with opposite spin polarizations. When $\lambda>0$, then in contrast with the case of $U^{\prime}=0$, the phonon satellites of the both Andreev bound states appear on one side of each pair of the main DOS maxima. The minima between each pair of the Andreev resonances are separated by the phonon energy. The result displayed in Fig. 1 shows also that the presence of the phonon mode gives rise to a slight DOS enhancement of the main AR resonances, while the on-dot repulsion does not affect the DOS intensity.

The effect of the electron-phonon interaction strength on the AR transmission through the F-QD-S system with interacting QD is illustrated in Fig. 2. First, an enhancement of the phonon side resonances due to the Franck Condon blockade with increasing $\lambda$ is predicted to appear (compare the dashed and dotted lines in Fig. 2). Second, with increasing quantity $\lambda$ the renormalization of the in-gap QD states occurs. Thus, in terms of energy, the distance between the Andreev bound states diminishes (see inset in Fig. 2 for details).

\section{Conclusions}

Using the NGF approach we have considered spinpolarized transport, associated with Andreev reflection, through a single-level quantum dot coupled to one $\mathrm{F}$ and one $\mathrm{S}$ leads. Features of the AR spectral function due to the Coulomb correlations on the dot, competing with the local phonon mode, were analyzed. Numerical results revealed that the phonon-assisted AR transmission through an interacting QD gives rise to such effects as phonon emission side bands (at assumed $T=0$ ) of the both Andreev bound states, enhancement of the main AR resonances, the $\lambda$-induced enhancement of the phonon satellite peaks in the DOS spectrum as well as the $\lambda$ dependent renormalization of the Andreev bound states.

\section{Acknowledgments}

This work was supported by the Polish Ministry of Science and Higher Education as a research project in the years 2010-2013.

\section{References}

[1] Y. Zhu, Q.-F. Sun, T.-H. Lin, Phys. Rev. B 65, 024516 (2001).

[2] Z.Y. Zeng, B. Li, F. Claro, Phys. Rev. B 68, 115319 (2003).

[3] X. Cao, Y. Shi, X. Song, S. Zhou, Phys. Rev. B 70, 235341 (2004).

[4] P. Zhang, Y. Li, J. Phys.: Condens Matter 21, 095602 (2009).

[5] K. Bocian, W. Rudziński, European Physical Journal Web of Conferences 40, 10002 (2013).

[6] G.D. Mahan, Many-Particle Physics, Plenum Press, New York 2000. 